\title{
ARTICLE
}

\section{Online and mobile customer behaviour: a critical evaluation of Grounded Theory studies}

\author{
Aikaterini C. Valvi \\ Department of Management, Birkbeck, University of London, Clore Management Centre, \\ Torrington Square, London, WC1E 7JL , UK
}

Constantinos C. Frangos

Division of Medicine, University College London , 21 University Street, London, WC1E 6JJ , UK, constantinos.frangos.09@ucl.ac.uk

Christos C. Frangos

Department of Business Administration, Technological Educational Institute (TEI) of Athens, 7 Tepeleniou Street, Iraklion Attikis 14121, Athens, Greece

\begin{abstract}
With the rapid increase in electronic and mobile commerce over the last few years, the academic literature on online and mobile customer behaviour has been fairly plentiful with a great deal of quantitative studies testing variations of existing customer behaviour theories. However, little attention has been given to qualitative studies in the field, which seek to explore new aspects of online or mobile customer behaviour, adding to existing theories or even creating new ones. Thus, the purpose of the present paper is to critically evaluate studies employing Grounded Theory (GT), a method commonly used for theory building in qualitative social research. Nine studies were identified examining online or mobile customer behaviour under this approach, providing theories based on emerging categories. Results of their studies seem to be very similar to existing customer behaviour theories, occasionally adding new categories to the existing theory nomenclature. Studies presented weaknesses regarding the accurate methodological conduct of GT and the process of generating theory, attributed predominantly to methodological, verification and reporting bias. Nevertheless, the main advantage of GT studies remains the generation of theory that can be applied in practice, reinforced by the presentation of conceptual prospects for testing new variables in quantitative studies. Overall, the contribution of GT studies to online and mobile customer behaviour research should be based on more rigorous methodology and aim to challenge rather than confirm existing theories with the purpose of advancing knowledge in the field.
\end{abstract}

Keywords: online customer behaviour, mobile customer behaviour, Grounded Theory, customer behaviour theory 


\section{Introduction}

The rapid expansion of the Internet from the 1990s onwards led to the development of electronic commerce (e-commerce), which has substantially changed the business landscape and the way customers perform online purchases through computer networks (Ngai and Gunasekaran 2007, Lu and Su 2009). Now, the phenomenon of online purchasing has been adopted by almost everyone (Abdul-Muhmin 2011) and it continues to find fertile ground with the advent of mobile commerce (m-commerce or wireless e-commerce) as customers can have access to the Internet via their mobile devices 'on an anywhere, anytime basis' (Balasubramanian et al. 2002, Park et al. 2008, Rose et al. 2011). Hence, identifying and understanding the potential drivers of online purchase behaviour has become an interesting research area in various research domains with an increasing number of publications every year (Grant et al. 2007) (Figure 1).

Online customer behaviour is currently examined under traditional customer behaviour theories; the Theory of Reasoned Action (Fishbein and Ajzen 1975), the Theory of Planned Behaviour (TPB) (Ajzen 1991), the Technology Acceptance Model (TAM) (Davis et al. 1989), the Innovation Diffusion Theory (IDT) (Rogers 1962), and the Expectation-Confirmation Theory (Bhattacherjee 2001) are the most frequently cited, giving the impression that there is no distinction between online and offline customer behaviour (Constantinides 2004, Jiang and Rosenbloom 2005).

However, a systematic review has identified that the scope of online customer behaviour studies is broad, disjointed and contradictory (Cheung et al. 2003). This may have resulted in a call for the development of new theories, an effort which has not yet been undertaken cohesively. Researchers should try to explore and build their own theories in online customer behaviour instead of applying existing ones (Cheung et al. 2003, p. 205), and in the words of Ranaweera et al. (2005, p. 52), 'online customer behaviour is still in an exploratory stage'.

Page 656 - - >

Figure 1. Number of articles on online consumer behaviour from Web of Knowledge.

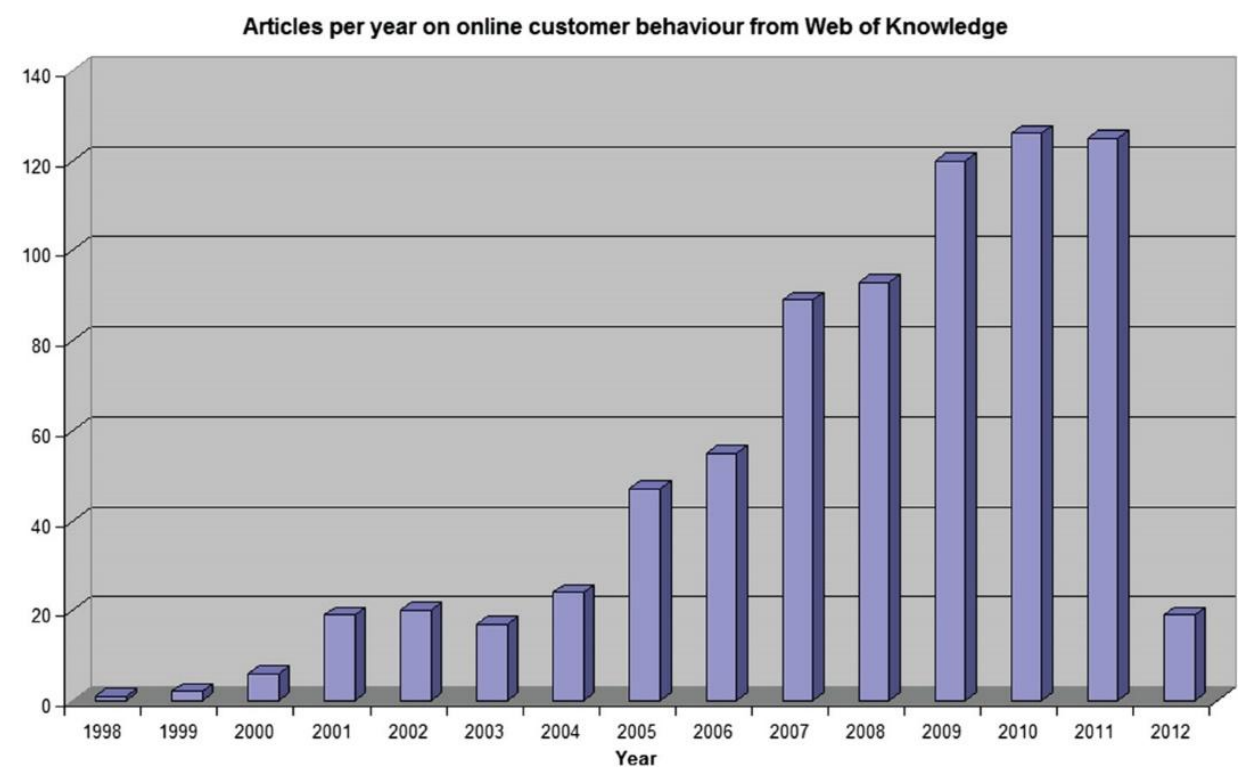


As a result, researchers in online customer behaviour need to deliberate on the use of theory testing and consider theory building by applying qualitative techniques. In the present paper, we focus on one method, Grounded Theory (GT), which has an ongoing interest and acceptance in business and management research (Goulding 2002, 2009, Jones and Noble 2007, Wagner et al. 2010). Nevertheless, critiques have been made of some texts regarding consumer behaviour that suggest that GT application in business has been executed with certain misconceptions regarding application of the method and taxonomy of epistemological background (Martin 2005).

These misnomers create the possibility of erroneous GT application in online and mobile consumer behaviour research. Hence, we intend to critically review the current body of GT research within the online/mobile customer behaviour literature by identifying possible principal constructs and agents of online/mobile customer behaviour. Also, the potential contribution of GT to existing customer behaviour theories, the methodological assessment of existing research, and future research implications in terms of such advances will be discussed.

\section{Grounded Theory}

GT is a research methodology that facilitates 'the discovery of theory from data' (Glaser and Strauss 1967, p. 1) with the aim of conceptualising the main concern of participants and how they resolve or process that concern rather than using a preconceived theory (Strauss and Corbin 1994, Goulding 2009). Specifically, it is a systematic yet flexible methodology for the concurrent collection and analysis of all types of data, including qualitative data, to construct theories that are grounded in data themselves (Glaser 1998, Glaser and Holton 2004, Charmaz 2006, p. 2). Since the original discovery in 1967 (Glaser and Strauss 1967), its use has become widespread but has also led to considerable debate on how to optimally attain GT (Hallberg 2006), and its ontology and theoretical viewpoints have been the topics of polemic (Bowen 2009, Chen and Boore 2009, Dunne 2011). Hence, there are three basic approaches to GT.

The first is classic GT, which is based on the original text of 1967 and the latter writings of Glaser $(1978,1998,2002 a, 2002 b, 2004)$. GT is a precise methodology underpinned by the constant comparative method and conceptualisations. Ideally, a GT researcher should initially ignore all the existing literature concerning the actual research area and just enter the research field attentively and with as few preconceptions as possible. Through constant comparisons of different data, categories will emerge on the part of the researcher, and thus theory 'will be a transcending abstraction' (Glaser 2002b). Classic GT does not fit within established research paradigms whether positivist, interpretivist, postmodern, or otherwise; rather, as a general methodology, it transcends the specific boundaries of established paradigms to accommodate any type of data sourced and expressed through any epistemological lens. Glaser (1998) views GT as a neutral tool which can be used by any researcher independent of their ontological/epistemological perspectives. What differentiates classic GT from other versions is the emphasis on conceptualisation and theory generation. It is based on conceptualisation not positivism (Glaser 2002a, 2002b).

Corbin and Strauss (1990) and Strauss and Corbin (1990) describe their different views of GT and its procedures, and this has been labelled the reformulated GT. Their view of GT seems to be more pragmatic than Glaser's also including a rejection of positivist positions. 
Corbin and Strauss (1990) explicitly argue that reality cannot be fully known but can always be interpreted. The relativist ontology is implicit when Strauss and Corbin claim that 'doing

Page 657 - - >

analysis is, in fact, making interpretations' (Strauss and Corbin 1990, p. 59). Thus, subjectivity enriches the research process because the generated theory is a created reality, constructed through a transactional process involving the researcher and the data (González-Teruel and Abad-García 2012). Strauss and Corbin's cooperation was critiqued by Glaser, who believed that the former had distorted the description of GT from its origins as a theory-generating method into being an entirely new method of full conceptual description (Glaser 2002b, 2005).

Finally, Charmaz $(1995,2000,2006)$ has presented a constructivist version of GT, which can be seen as an approach between positivism and postmodernism (Martin 2006a). Constructivism assumes that there are multiple simultaneous social realities, rather than one real reality (Hallberg 2006). In constructivist GT, data are constructed through an ongoing interaction between researcher and participant, where the actual meaning and reality are created during individuals' reflexive interactions in real settings (Gephart 2004, Charmaz 2006). The researcher's interpretative understanding, rather than the researcher's explanation, of how the participant creates his or her understanding and meaning of reality is the result of the analysis. This type of theory is in line with symbolic interactionism, and both view social life as process-driven and assume emergent multiple realities (Charmaz 2006). The result of a constructivist GT study is more seldom presented as a theory than as a story or a narrative about people, social processes, and situations.

A grounded theorist aims to saturate individual concepts in the pursuit of theoretical completeness (Glaser 2004, Glaser and Holton 2004). Theoretical completeness is achieved when the researcher has developed an 'integrated theory which accounts for most of the action in a substantive area' (Glaser 1998, p. 86). The emergence of a core category is essential for the integration of other categories into a theory. Theoretical sampling should occur throughout the GT process, aiming to develop new categories and saturate each emergent category. The analysis starts with line-by-line open coding and following recognition of the core category, continues with selective coding. The researcher's objective is to conceptualise, to develop categories and propose relationships between categories (Coyne 1997, p. 625, Suddaby 2006, Payne 2007, p. 68). This conceptualisation is carried out in memos which play an important role in GT analysis, as memos offer researchers the opportunity to note down ideas and expected associations for emerging categories.

\subsection{GT in customer behaviour research}

The first GT article in customer behaviour literature appeared in 1989 (Belk et al. 1989), followed by a number of others until today (Pettigrew 2002, Goulding 2005, Kim et al. 2009). Former reviews in customer behaviour research (Goulding 2005, Jones and Noble 2007, Wagner et al. 2010) depict the continuous interest of researchers in understanding customers' experiences and motivations among various marketing areas.

However, their findings cannot be immediately applied to the online environment, and even less to the mobile environment. Research in online customer behaviour has more than once 
shown its unique characteristics compared to traditional marketing and customer behaviour theories and has added new variables to existing models through plentiful quantitative studies (Chen et al. 2008, Valvi and Fragkos 2012). The first key difference is the involvement of information technology in accessing the virtual or mobile store and consequently performing purchasing behaviour (Koufaris 2002). The introduction of such advanced technologies has introduced technologically related predictors of customer behaviour, and this list keeps becoming more extensive in the present mobile era (Yuan and Zhang 2003, Park and Gretzel 2007, Wu et al. 2010). Furthermore, in an online or mobile experience, personal contact is decreased in comparison with an offline customer experience; information provision is intensive; brand presentation is audiovisual, compared to tangible devices offline; and time for interactions is consumer dictated (Rose et al. 2011).

Hence, exploratory research with qualitative techniques is necessitated in view of the unique traits of online and mobile technologies. Application of the GT method is in proximate need of assessment, since its methodology already presents constant variations, contradictions, and modifications (Jones and Noble 2007). The starting point of the present research will be the identification of GT's evaluative criteria in online/mobile customer behaviour, in order to distinguish the fundamental elements (dependability, credibility, transferability, and confirmability) that make GT research trustworthy, accurate, verifiable, and rigorous (Creswell and Miller 2000).

\section{Methods}

\subsection{Evaluation criteria}

The characteristics of a GT approach that have been identified within the online customer behaviour domain are the same as those mentioned above: simultaneous data collection, coding, and analysis; determination of the core and emerging categories; use of the constant comparison process and conceptualisation; and theoretical sampling facilitating new data to emerge until theoretical completeness ensues. Using these criteria and answering the list of questions developed by Charmaz (2006) and Hutchison et al. (2011) (Table 1), we intend to assess the quality of each reviewed study.

Table 1. Questions to guide the extraction of study quality related information (Hutchison et al. 2011, p. 252).

\footnotetext{
-Is GT an appropriate methodology for this research?

Was a justification presented for adopting a GT approach? If so what was it?

How was GT defined? (e.g. as a research methodology or simply a data analysis tool?)

-Was sampling conducted in accordance with the tenets of GT?

What evidence is there to suggest that sampling was conducted to facilitate theory generation?

How was the initial sample selected? On what grounds?

Did theoretical formulations guide some of the data collection, if so how?

Based on the answers to the above two questions did theoretical sampling occur?

Is there evidence of concurrent involvement in data collection and analysis?

-How were the initial concepts and categories developed?

What initial concepts and categories were presented?

What techniques were used to construct or develop these categories (coding, memo writing, comparisons, questioning, use of attributes, etc.)?

What evidence is there to suggest that these concepts or categories were generated from the data itself and not from pre-conceived logically deduced hypotheses?

Do the initial categories cover a wide range of empirical observations, was the initial focus
} 
broad?

How did theoretical development continue after the initial concept identification?

How did theory development advance during each step of data collection and analysis?

What major categories were presented?

What techniques were used to construct or develop these categories (e.g. axial or focused coding, systematic comparisons, questioning)?

What evidence is there to suggest that the constant comparison method was used? That is, were systematic comparisons made between observations and between categories?

Are the categories theoretically dense? Are there clear links between individual categories and subcategories as well as between individual categories and the larger core category? Have the dimensions of categories and subcategories been explored?

-What was the end product of this research and how was it finally constructed?

How and why was the core category selected? On what grounds were the final analytical decisions made?

What evidence is there to suggest that the study achieved theoretical saturation?

What conclusions were drawn?

What evidence is there to suggest that the results offer new insight into the studied phenomenon?

Page $658-->$

\subsection{Sources}

A systematic literature review of GT research within online/mobile customer behaviour literature has been undertaken by using the following databases: Web of Knowledge, Scopus, Business Source Premier, ABI Inform, and Google Scholar. A number of different keyword combinations were used by including 'Grounded Theory and Online Customer/Consumer/Buyer Behaviour'; 'Grounded Theory and Online Behaviour'; 'Grounded Theory and Internet Behaviour'; 'Grounded Theory and Online Purchase'; and 'Grounded Theory and Online Shopping'. 


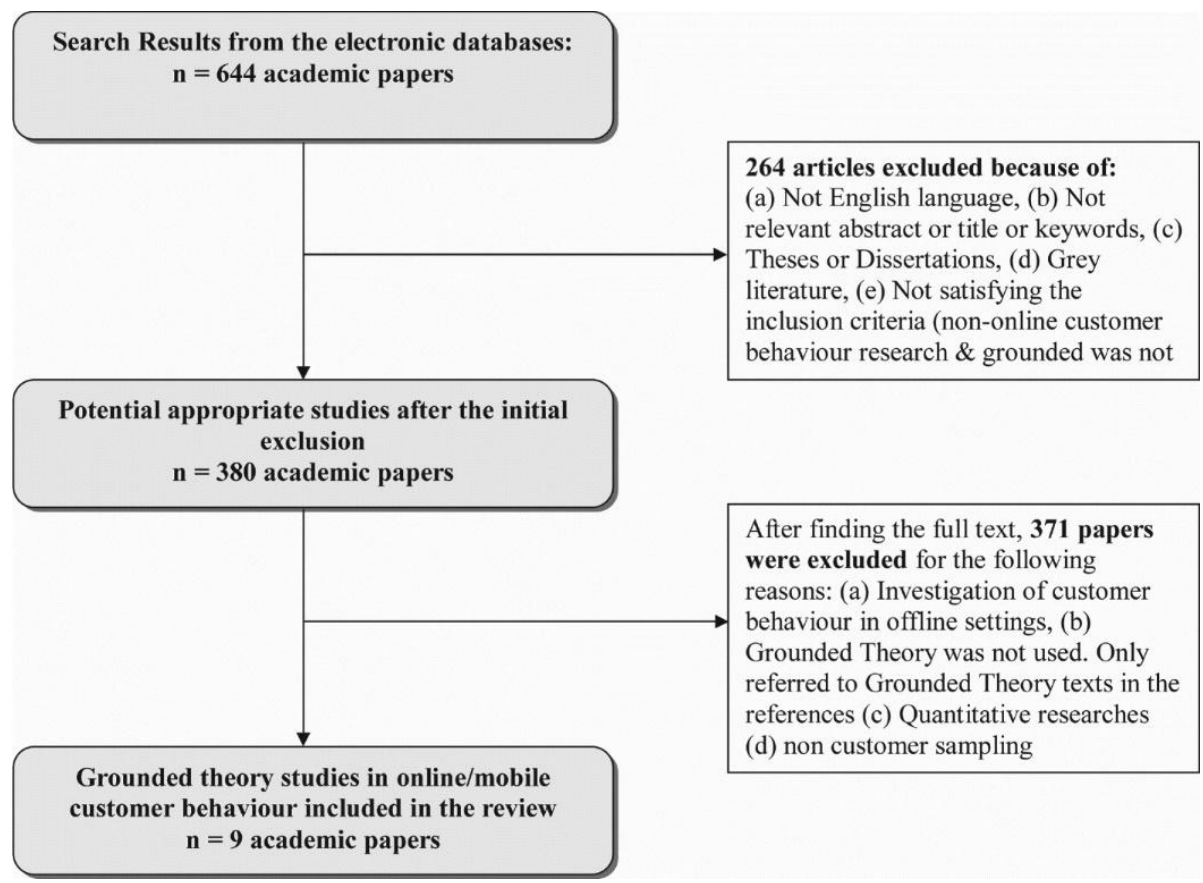

Figure 2. Study selection process.

Page 659 - -> 
Table 2. Characteristics of reviewed GT studies.

\begin{tabular}{|c|c|c|c|c|c|c|c|c|c|c|}
\hline & $\begin{array}{l}\text { Ref } \\
\text { ere } \\
\text { nc } \\
\text { e }\end{array}$ & Purpose & $\begin{array}{l}\text { Data } \\
\text { collecti } \\
\text { on }\end{array}$ & Participants & \begin{tabular}{|l|}
$\mathbf{M}$ \\
$\mathrm{e}$ \\
$\mathrm{m}$ \\
$\mathbf{o}$ \\
$\mathrm{i}$ \\
$\mathrm{n}$ \\
$\mathrm{g}$
\end{tabular} & $\begin{array}{l}\text { Sam } \\
\text { pling }\end{array}$ & Core category & Emerging categories & \begin{tabular}{|l|} 
GT \\
ver \\
sio \\
n
\end{tabular} & $\begin{array}{l}\text { Categ } \\
\text { ories' } \\
\text { simila } \\
\text { rity } \\
\text { with } \\
\text { existin } \\
\text { g } \\
\text { custo } \\
\text { mer } \\
\text { behavi } \\
\text { our } \\
\text { theori } \\
\text { es }\end{array}$ \\
\hline 1 & \begin{tabular}{|l} 
Lic \\
hte \\
nst \\
ein \\
an \\
d \\
Will \\
iam \\
son \\
$(20$ \\
$06)$ \\
\end{tabular} & $\begin{array}{l}\text { To explore } \\
\text { the factors } \\
\text { affecting } \\
\text { Australian } \\
\text { consumer } \\
\text { s in the } \\
\text { adoption } \\
\text { of Internet } \\
\text { banking }\end{array}$ & $\begin{array}{l}\text { Semi- } \\
\text { structur } \\
\text { ed } \\
\text { intervie } \\
\text { ws } \\
\text { (individ } \\
\text { ual and } \\
\text { focus } \\
\text { group } \\
\text { intervie } \\
\text { ws) }\end{array}$ & $\begin{array}{l}32 \\
\text { Australians }\end{array}$ & $\begin{array}{l}N \\
0\end{array}$ & $\begin{array}{l}\text { Both } \\
\text { purp } \\
\text { osive } \\
\text { and } \\
\text { theor } \\
\text { etical } \\
\text { sam } \\
\text { pling } \\
\text { were } \\
\text { used }\end{array}$ & $\begin{array}{l}\text { Not clear. Internet baking } \\
\text { adoption was the main concern }\end{array}$ & $\begin{array}{l}\text { Not clear. attention, accessibility, } \\
\text { convenience, self-efficacy, } \\
\text { usability, risks and costs, relative } \\
\text { advantage, knowledge and } \\
\text { support }\end{array}$ & $\begin{array}{l}\text { Ref } \\
\text { or } \\
\text { mul } \\
\text { ate } \\
\text { d } \\
\text { GT; } \\
\text { con } \\
\text { stru } \\
\text { ctiv } \\
\text { ist } \\
\text { GT } \\
\end{array}$ & $\begin{array}{l}\text { TAM, } \\
\text { TPB }\end{array}$ \\
\hline
\end{tabular}




\begin{tabular}{|c|c|c|c|c|c|c|c|c|c|c|}
\hline $\begin{array}{l}\mathrm{N} \\
\mathbf{0} \\
.\end{array}$ & $\begin{array}{l}\text { Ref } \\
\text { ere } \\
\text { nc } \\
\text { e }\end{array}$ & Purpose & $\begin{array}{l}\text { Data } \\
\text { collecti } \\
\text { on }\end{array}$ & Participants & \begin{tabular}{l|}
$\mathbf{M}$ \\
$\mathbf{e}$ \\
$\mathbf{m}$ \\
$\mathbf{0}$ \\
$\mathbf{i}$ \\
$\mathbf{n}$ \\
$\mathbf{g}$
\end{tabular} & $\begin{array}{l}\text { Sam } \\
\text { pling }\end{array}$ & Core category & Emerging categories & $\begin{array}{l}\text { GT } \\
\text { ver } \\
\text { sio } \\
\text { n }\end{array}$ & $\begin{array}{l}\text { Categ } \\
\text { ories' } \\
\text { simila } \\
\text { rity } \\
\text { with } \\
\text { existin } \\
\text { g } \\
\text { custo } \\
\text { mer } \\
\text { behavi } \\
\text { our } \\
\text { theori } \\
\text { es }\end{array}$ \\
\hline 2 & $\begin{array}{l}\text { Da } \\
\text { en } \\
\text { gb } \\
\text { up } \\
\text { ph } \\
\text { aet } \\
\text { al. } \\
(20 \\
06)\end{array}$ & $\begin{array}{l}\text { To explore } \\
\text { visitor } \\
\text { experienc } \\
\text { es at } \\
\text { heritage } \\
\text { sites }\end{array}$ & $\begin{array}{l}\text { In- } \\
\text { depth } \\
\text { intervie } \\
\text { ws, } \\
\text { observ } \\
\text { ations } \\
\text { and } \\
\text { publish } \\
\text { ed } \\
\text { materia } \\
\text { I and } \\
\text { archive } \\
\text { data }\end{array}$ & $\begin{array}{l}\text { The personal } \\
\text { profiles or } \\
\text { details of the } \\
\text { participants } \\
\text { were not } \\
\text { used in the } \\
\text { analysis and } \\
\text { have not } \\
\text { been } \\
\text { presented in } \\
\text { reports of the } \\
\text { study }\end{array}$ & $\begin{array}{l}\mathrm{Y} \\
\mathrm{e} \\
\mathrm{s}\end{array}$ & $\begin{array}{l}\text { Theo } \\
\text { retic } \\
\text { al }\end{array}$ & Interactive experience & $\begin{array}{l}\text { Matter of choice, perceived value } \\
\text { of heritage, involvement, } \\
\text { perception of authenticity, quest of } \\
\text { experience, visitor practices, } \\
\text { evaluation of experience, } \\
\text { conservation moral, behavioural } \\
\text { norms, acquisition of knowledge }\end{array}$ & $\begin{array}{l}\text { Ref } \\
\text { or } \\
\text { mul } \\
\text { ate } \\
\text { d } \\
\text { GT }\end{array}$ & $\begin{array}{l}\text { TPB, } \\
\text { TAM }\end{array}$ \\
\hline
\end{tabular}




\begin{tabular}{|c|c|c|c|c|c|c|c|c|c|c|}
\hline & $\begin{array}{l}\text { Ref } \\
\text { ere } \\
\text { nc } \\
\text { e }\end{array}$ & Purpose & $\begin{array}{l}\text { Data } \\
\text { collecti } \\
\text { on }\end{array}$ & Participants & \begin{tabular}{|l|}
$\mathbf{M}$ \\
$\mathrm{e}$ \\
$\mathrm{m}$ \\
$\mathbf{o}$ \\
$\mathrm{i}$ \\
$\mathrm{n}$ \\
$\mathrm{g}$ \\
\end{tabular} & $\begin{array}{l}\text { Sam } \\
\text { pling }\end{array}$ & Core category & Emerging categories & \begin{tabular}{|l|} 
GT \\
ver \\
sio \\
n \\
\end{tabular} & $\begin{array}{l}\text { Categ } \\
\text { ories' } \\
\text { simila } \\
\text { rity } \\
\text { with } \\
\text { existin } \\
\text { g } \\
\text { custo } \\
\text { mer } \\
\text { behavi } \\
\text { our } \\
\text { theori } \\
\text { es }\end{array}$ \\
\hline 3 & $\begin{array}{l}\mathrm{Na} \\
\mathrm{mk} \\
\text { un } \\
\mathrm{g} \mathrm{e} \\
\mathrm{t} \text { al. } \\
(20 \\
07)\end{array}$ & $\begin{array}{l}\text { To } \\
\text { understan } \\
d \text { the } \\
\text { Website } \\
\text { experienc } \\
\text { e of } \\
\text { restaurant } \\
\text { customers }\end{array}$ & $\begin{array}{l}\text { In- } \\
\text { depth } \\
\text { semi- } \\
\text { structur } \\
\text { ed } \\
\text { intervie } \\
\text { ws }\end{array}$ & $\begin{array}{l}37 \text { web } \\
\text { restaurant } \\
\text { customers }\end{array}$ & \begin{tabular}{|l|}
$Y$ \\
$e$ \\
S
\end{tabular} & $\begin{array}{l}\text { Theo } \\
\text { retic } \\
\text { al }\end{array}$ & $\begin{array}{l}\text { More than one core category } \\
\text { were found (source of } \\
\text { information, aim of visiting } \\
\text { Websites, Website experience, } \\
\text { Website usability, } \\
\text { demographics, socio- } \\
\text { psychographics, webographics, } \\
\text { attracting Internet users, } \\
\text { building relationship, level of } \\
\text { communication, formed } \\
\text { relationship, effect of building } \\
\text { relationship) }\end{array}$ & $\begin{array}{l}\text { A number of sub categories for } \\
\text { each core category was found. } \\
\text { Some of the sub categories were: } \\
\text { word of mouth, goal oriented, } \\
\text { frequently visited Website, } \\
\text { motivation for visiting Website, } \\
\text { information, interactivity, family life } \\
\text { cycle, purpose of Internet usage, } \\
\text { Website experience, eating } \\
\text { behaviour, individualisation, } \\
\text { company's and customer's } \\
\text { perspective }\end{array}$ & $\begin{array}{l}\text { Ref } \\
\text { or } \\
\text { mul } \\
\text { ate } \\
\text { d } \\
\text { GT }\end{array}$ & TAM \\
\hline
\end{tabular}




\begin{tabular}{|c|c|c|c|c|c|c|c|c|c|c|}
\hline & $\begin{array}{l}\text { Ref } \\
\text { ere } \\
\text { nc } \\
\text { e }\end{array}$ & Purpose & $\begin{array}{l}\text { Data } \\
\text { collecti } \\
\text { on }\end{array}$ & Participants & \begin{tabular}{|l}
$\mathbf{M}$ \\
$\mathrm{e}$ \\
$\mathrm{m}$ \\
$\mathbf{0}$ \\
$\mathbf{i}$ \\
$\mathbf{n}$ \\
$\mathbf{g}$
\end{tabular} & $\begin{array}{l}\text { Sam } \\
\text { pling }\end{array}$ & Core category & Emerging categories & \begin{tabular}{|l|} 
GT \\
ver \\
sio \\
n \\
\end{tabular} & $\begin{array}{l}\text { Categ } \\
\text { ories' } \\
\text { simila } \\
\text { rity } \\
\text { with } \\
\text { existin } \\
\text { g } \\
\text { custo } \\
\text { mer } \\
\text { behavi } \\
\text { our } \\
\text { theori } \\
\text { es }\end{array}$ \\
\hline 4 & $\begin{array}{l}\text { Ma } \\
\text { dell } \\
\text { an } \\
d \\
\text { Mu } \\
\text { nce } \\
r \\
(20 \\
07)\end{array}$ & $\begin{array}{l}\text { To } \\
\text { understan } \\
d \text { why } \\
\text { young } \\
\text { people } \\
\text { use the } \\
\text { Internet } \\
\text { and } \\
\text { mobile } \\
\text { phones for } \\
\text { social } \\
\text { purposes }\end{array}$ & $\begin{array}{l}\text { Focus } \\
\text { groups }\end{array}$ & $\begin{array}{l}\text { In each } \\
\text { group, } 6 \text { of } \\
\text { the } \\
\text { participants } \\
\text { were female } \\
\text { and } 1 \text { was } \\
\text { male (total: } \\
14 \text { students- } \\
\text { users) }\end{array}$ & $\begin{array}{l}\mathrm{N} \\
\mathrm{O}\end{array}$ & $\begin{array}{l}\text { Not } \\
\text { repor } \\
\text { ted }\end{array}$ & Control over social interactions & Not reported & \begin{tabular}{|l} 
Cla \\
ssi \\
C \\
GT
\end{tabular} & TPB \\
\hline & & $\begin{array}{l}\text { Page } \\
660 \rightarrow\end{array}$ & & & & & & & & \\
\hline
\end{tabular}




\begin{tabular}{|c|c|c|c|c|c|c|c|c|c|c|}
\hline & $\begin{array}{l}\text { Ref } \\
\text { ere } \\
\text { nc } \\
\text { e }\end{array}$ & Purpose & $\begin{array}{l}\text { Data } \\
\text { collecti } \\
\text { on }\end{array}$ & Participants & \begin{tabular}{|l|}
$\mathrm{M}$ \\
$\mathrm{e}$ \\
$\mathrm{m}$ \\
$\mathbf{0}$ \\
$\mathrm{i}$ \\
$\mathrm{n}$ \\
$\mathrm{g}$
\end{tabular} & $\begin{array}{l}\text { Sam } \\
\text { pling }\end{array}$ & Core category & Emerging categories & \begin{tabular}{|l} 
GT \\
ver \\
sio \\
$\mathbf{n}$
\end{tabular} & $\begin{array}{l}\text { Categ } \\
\text { ories' } \\
\text { simila } \\
\text { rity } \\
\text { with } \\
\text { existin } \\
\text { g } \\
\text { custo } \\
\text { mer } \\
\text { behavi } \\
\text { our } \\
\text { theori } \\
\text { es }\end{array}$ \\
\hline 5 & $\begin{array}{l}\text { An } \\
\text { dre } \\
\text { ws } \\
\text { an } \\
\text { d } \\
\text { Bo } \\
\text { yle } \\
(20 \\
08)\end{array}$ & $\begin{array}{l}\text { To extend } \\
\text { marketing } \\
\text { knowledge } \\
\text { into } \\
\text { perceived } \\
\text { risk in } \\
\text { online } \\
\text { transactio } \\
\text { ns }\end{array}$ & $\begin{array}{l}\text { In- } \\
\text { depth } \\
\text { semi- } \\
\text { structur } \\
\text { e } \\
\text { intervie } \\
\text { ws }\end{array}$ & $\begin{array}{l}10 \\
\text { interviewees } \\
\text { with internet } \\
\text { and online } \\
\text { purchasing } \\
\text { experience }\end{array}$ & $\begin{array}{l}N \\
o \\
t \\
c \\
I \\
e \\
a \\
r\end{array}$ & $\begin{array}{l}\text { Both } \\
\text { purp } \\
\text { osive } \\
\text { and } \\
\text { theor } \\
\text { etical } \\
\text { sam } \\
\text { pling } \\
\text { were } \\
\text { used }\end{array}$ & $\begin{array}{l}\text { Influence of communication } \\
\text { sources in the diffusion of } \\
\text { online purchasing. }\end{array}$ & $\begin{array}{l}\text { Human manipulation of Internet } \\
\text { technology, subjective/emotive, } \\
\text { objective/rational, paranoid, credit } \\
\text { card fraud, computer system } \\
\text { invasion, mass media influence } \\
\text { on perceptions of risk online, } \\
\text { inter-personal communication, } \\
\text { intra-personal communication }\end{array}$ & $\begin{array}{l}\text { Ref } \\
\text { or } \\
\text { mul } \\
\text { ate } \\
\text { d } \\
\text { GT }\end{array}$ & $\begin{array}{l}\text { TAM, } \\
\text { IDT }\end{array}$ \\
\hline 6 & $\begin{array}{l}\text { Hal } \\
\text { aw } \\
\text { eh } \\
\text { et } \\
\text { al. } \\
(20 \\
08)\end{array}$ & $\begin{array}{l}\text { To explore } \\
\text { customers' } \\
\text { perception } \\
\text { in e- } \\
\text { commerce } \\
\text { Websites } \\
\text { in Jordan }\end{array}$ & $\begin{array}{l}\text { Intervie } \\
\text { ws }\end{array}$ & 10 customers & \begin{tabular}{|l|}
$N$ \\
0
\end{tabular} & $\begin{array}{l}\text { Not } \\
\text { repor } \\
\text { ted }\end{array}$ & Psychological aspect of security & Not reported & $\begin{array}{l}\text { Ref } \\
\text { or } \\
\text { mul } \\
\text { ate } \\
\text { d } \\
\text { GT }\end{array}$ & $\begin{array}{l}\text { TAM, } \\
\text { TPB }\end{array}$ \\
\hline
\end{tabular}




\begin{tabular}{|c|c|c|c|c|c|c|c|c|c|c|}
\hline & $\begin{array}{l}\text { Ref } \\
\text { ere } \\
\text { nc } \\
\text { e }\end{array}$ & Purpose & $\begin{array}{l}\text { Data } \\
\text { collecti } \\
\text { on }\end{array}$ & Participants & $\begin{array}{l} \\
\text { e } \\
\text { m } \\
o \\
i \\
n \\
g\end{array}$ & $\begin{array}{l}\text { Sam } \\
\text { pling }\end{array}$ & Core category & Emerging categories & $\begin{array}{l}\text { GT } \\
\text { ver } \\
\text { sio } \\
\text { n }\end{array}$ & $\begin{array}{l}\text { Categ } \\
\text { ories' } \\
\text { simila } \\
\text { rity } \\
\text { with } \\
\text { existin } \\
\text { g } \\
\text { custo } \\
\text { mer } \\
\text { behavi } \\
\text { our } \\
\text { theori } \\
\text { es }\end{array}$ \\
\hline 7 & $\begin{array}{l}\text { Bru } \\
\text { ng } \\
\text { er } \\
\text { an } \\
\text { d } \\
\text { Per } \\
\text { elli } \\
(20 \\
09)\end{array}$ & $\begin{array}{l}\text { To } \\
\text { understan } \\
\mathrm{d} \text { the } \\
\text { behaviour } \\
\text { of } \\
\text { customers } \\
\text { who use } \\
\text { travel } \\
\text { Internet } \\
\text { Websites }\end{array}$ & $\begin{array}{l}\text { Face- } \\
\text { to-face } \\
\text { and } \\
\text { telepho } \\
\text { ne } \\
\text { intervie } \\
\text { ws }\end{array}$ & $\begin{array}{l}15 \\
\text { 'experienced' } \\
\text { online } \\
\text { travellers }\end{array}$ & $\begin{array}{l}N \\
0\end{array}$ & $\begin{array}{l}\text { Purp } \\
\text { osive } \\
\text { (non- } \\
\text { rand } \\
\text { om } \\
\text { sam } \\
\text { ple) }\end{array}$ & Not reported & $\begin{array}{l}\text { Changes in search mechanics, } \\
\text { breadth, involvement, control and } \\
\text { social interaction, issues and } \\
\text { respondent-designated } \\
\text { demographic categories }\end{array}$ & $\begin{array}{l}\text { Cla } \\
\text { ssi } \\
\text { C } \\
\text { GT }\end{array}$ & TPB \\
\hline 8 & $\begin{array}{l}\text { Pal } \\
\text { ka } \\
\text { et } \\
\text { al. } \\
(20 \\
09)\end{array}$ & $\begin{array}{l}\text { To explore } \\
\text { customers' } \\
\text { motivation } \\
\text { s, attitudes } \\
\text { and } \\
\text { behaviour } \\
\text { s of mobile } \\
\text { viral } \\
\text { marketing }\end{array}$ & $\begin{array}{l}\text { Focus } \\
\text { groups } \\
\text { and in- } \\
\text { depth } \\
\text { intervie } \\
\text { ws }\end{array}$ & $\begin{array}{l}57 \text { German } \\
\text { consumers }\end{array}$ & $\begin{array}{l}\mathrm{N} \\
\mathrm{O}\end{array}$ & $\begin{array}{l}\text { Theo } \\
\text { retic } \\
\text { al }\end{array}$ & $\begin{array}{l}\text { Three different models were } \\
\text { used (receipt, usage, } \\
\text { forwarding). The core category } \\
\text { for each model was intention to } \\
\text { open, intention to use, intention } \\
\text { to forward }\end{array}$ & $\begin{array}{l}\text { A number of emerging categories } \\
\text { were found based on social, } \\
\text { attitudinal, resource-based, } \\
\text { consumption-based, personal and } \\
\text { causal conditions as well as } \\
\text { auction/interaction strategy }\end{array}$ & $\begin{array}{l}\text { Ref } \\
\text { or } \\
\text { mul } \\
\text { ate } \\
\text { d } \\
\text { GT }\end{array}$ & $\begin{array}{l}\text { TAM, } \\
\text { TPB }\end{array}$ \\
\hline
\end{tabular}




\begin{tabular}{|c|c|c|c|c|c|c|c|c|c|c|}
\hline $\bar{N}$ & $\begin{array}{l}\text { Ref } \\
\text { ere } \\
\text { nc } \\
\text { e }\end{array}$ & Purpose & $\begin{array}{l}\text { Data } \\
\text { collecti } \\
\text { on }\end{array}$ & Participants & \begin{tabular}{|l|}
$M$ \\
$\mathbf{e}$ \\
$\mathbf{m}$ \\
$\mathbf{o}$ \\
$\mathbf{i}$ \\
$\mathbf{n}$ \\
$\mathbf{g}$
\end{tabular} & $\begin{array}{l}\text { Sam } \\
\text { pling }\end{array}$ & Core category & Emerging categories & \begin{tabular}{|l|} 
GT \\
ver \\
sio \\
n \\
\end{tabular} & $\begin{array}{l}\text { Categ } \\
\text { ories' } \\
\text { simila } \\
\text { rity } \\
\text { with } \\
\text { existin } \\
\text { g } \\
\text { custo } \\
\text { mer } \\
\text { behavi } \\
\text { our } \\
\text { theori } \\
\text { es }\end{array}$ \\
\hline 9 & \begin{tabular}{|l} 
Pa \\
pat \\
ha \\
nas \\
sis \\
an \\
$d$ \\
Kn \\
olle \\
$(20$ \\
$11)$
\end{tabular} & $\begin{array}{l}\text { To explore } \\
\text { user } \\
\text { perception } \\
\text { s and the } \\
\text { utilisation } \\
\text { of online } \\
\text { holiday } \\
\text { reviews for } \\
\text { holiday } \\
\text { purchase } \\
\text { decisions }\end{array}$ & $\begin{array}{l}\text { Observ } \\
\text { ation } \\
\text { and } \\
\text { intervie } \\
\text { ws }\end{array}$ & $\begin{array}{l}22 \text { online } \\
\text { users }\end{array}$ & $\begin{array}{l}N \\
0\end{array}$ & $\begin{array}{l}\text { Purp } \\
\text { osive }\end{array}$ & Online review utilisation & $\begin{array}{l}\text { Adoption readiness (content } \\
\text { richness, content accessibility), } \\
\text { source - heuristic assessment } \\
\text { (source micro-reputation, source } \\
\text { macro-reputation), content } \\
\text { heuristic assessment (perceived } \\
\text { realism, perceived neutrality, } \\
\text { negative bias, perceived } \\
\text { factuality) }\end{array}$ & $\begin{array}{l}\text { Cla } \\
\text { Ssi } \\
\text { C } \\
\text { GT; } \\
\text { ref } \\
\text { or } \\
\text { mul } \\
\text { ate } \\
\text { d } \\
\text { GT }\end{array}$ & TAM \\
\hline
\end{tabular}


Page 661 - - >

Searches followed a number of inclusion criteria. Only articles written in English and limited to management/marketing/advertising journals were included. Articles could be from conference proceedings or journals, but only records whose abstract was available were included (Rose et al. 2011). Dissertations, theses, and other materials from grey literature were excluded (Ridley 2008, Wallace and Wray 2011). We included studies that satisfied the following criteria: (a) online/mobile customer behaviour related research and (b) studies that explicitly stated the adoption of a GT methodology (Figure 2). Moreover, a snowball sampling technique was adopted by searching the references of the major review papers in order to ensure that all suitable papers were included (Park and Gretzel 2007).

\subsection{Procedure}

Hard copies of all publications that met the inclusion criteria were obtained. A comprehensive reading of those studies was followed in order to summarise the characteristics of each study identified (Table 2).

\section{Results}

\subsection{Study characteristics}

We identified nine GT studies in online/mobile customer behaviour research (seven online, one mobile customer behaviour, and one combination of both) based on different industries. Two papers per year were published in 2006, 2007, 2008, and 2009, and one paper in 2011. Sample sizes ranged from 10 to 57 customers, while in some cases the number of informants was not mentioned. Data were collected using in-depth semi-structured interviews, focus groups, or observations, and in some instances a combination of those. Although all studies explained the use of GT, only one justified the rationale of selection (Daengbuppha et al. 2006). Seven out of nine studies reported using GT throughout the entire study or recognised it as a research methodology, and only two studies reported using GT methods for analysing data (Madell and Muncer 2007, Halaweh et al. 2008). Six studies (Daengbuppha et al. 2006, Lichtenstein and Williamson 2006, Namkung et al. 2007, Andrews and Boyle 2008, Halaweh et al. 2008, Palka et al. 2009) described following reformulated GT by Strauss and Corbin (1990), two followed classic GT (Madell and Muncer 2007, Brunger and Perelli 2009), and one described following both (Papathanassis and Knolle 2011).

Figures 3 and 4 show 11 common characteristics that should be present in GT studies. All characteristics have at least once instance of not being described or their description being unclear from the paper. Nevertheless, the description of the core category was present in most studies. The characteristic least described was memoing, followed by theoretical completeness and constant comparisons. Coding was also inadequately described in the two classic GT studies, with only open coding described once. 


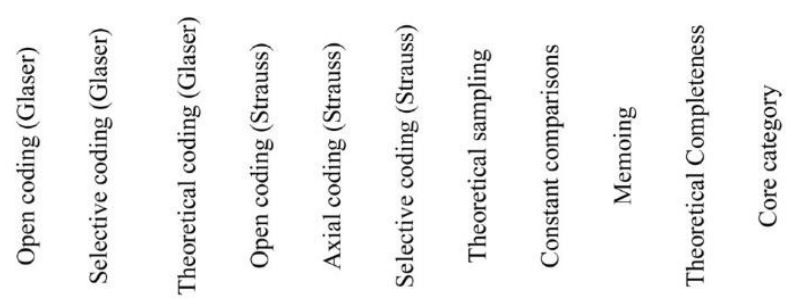

Lichtenstein and Williamson (2006)
Daengbuppha et al. (2006)
Namkung et al. (2007)
Madell and Muncer (2007)
Andrews and Boyle (2008)
Halaweh et al. (2008)
Brunger and Perelli (2009)
Palka et al. (2009)
Papathanassis and Knolle (2011)

\begin{tabular}{|c|c|c|c|c|c|c|c|c|c|c|}
\hline & & & $?$ & $?$ & ? & + & $\theta$ & $\theta$ & $\theta$ & $?$ \\
\hline & & & $\odot$ & + & + & $\odot$ & $\odot$ & 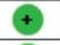 & $\odot$ & + \\
\hline & & & $?$ & $?$ & $?$ & + & + & + & $?$ & $?$ \\
\hline \multirow[t]{3}{*}{$?$} & $?$ & $?$ & & & & $?$ & $?$ & $\theta$ & $\theta$ & $\odot$ \\
\hline & & & $\odot$ & 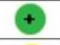 & $\odot$ & $\odot$ & $\odot$ & $?$ & $?$ & + \\
\hline & & & $?$ & $?$ & $?$ & $?$ & $\odot$ & $\theta$ & $\theta$ & + \\
\hline \multirow[t]{2}{*}{(} & $?$ & $?$ & & & & $\theta$ & $?$ & $\theta$ & $\odot$ & $\theta$ \\
\hline & & & 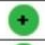 & + & + & $\odot$ & $?$ & $?$ & $\odot$ & + \\
\hline$\theta$ & $\theta$ & $\theta$ & $\odot$ & $\odot$ & $\odot$ & $\theta$ & $?$ & $\theta$ & $\odot$ & $\odot$ \\
\hline
\end{tabular}

$\odot$ : Present $\stackrel{?}{?}$ : Unclear $\odot$ : Not present

Figure 3. Use of GT procedures: review authors' judgements about each item for each included study.

Page 662 - - >

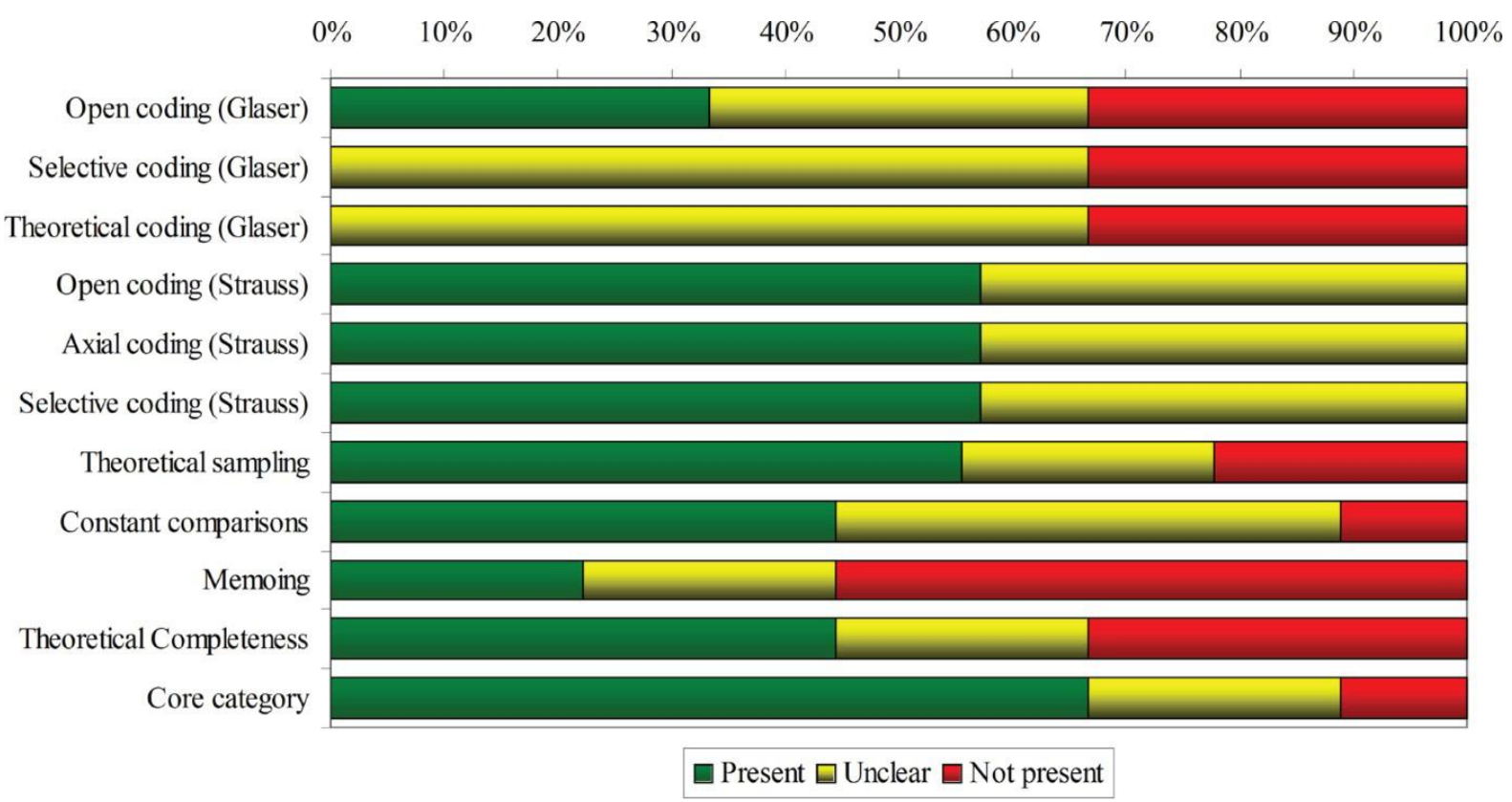

Figure 4. Use of GT procedures: review authors' judgements about each item presented as percentages across all included studies.

\subsection{Quality of the reviewed studies}

4.2.1. How were the initial concepts and categories developed?

The generation of initial concepts and categories was carried out by predominantly using coding techniques and less constant comparisons. While open coding was used by almost all studies (except from one), the extent to which this coding process involved selective, theoretical, or axial coding as well varied across studies. The use of memoing was 
inconsistent as it was only reported by two out of nine studies for theory development (Daengbuppha et al. 2006, Namkung et al. 2007).

\subsubsection{Was sampling conducted in accordance with the tenets of GT?}

Different initial sampling was selected from the studies. Three out of nine studies reviewed used theoretical sampling (Daengbuppha et al. 2006, Namkung et al. 2007, Palka et al. 2009), while two studies employed purposive sampling (Brunger and Perelli 2009, Papathanassis and Knolle 2011), presenting the rationale behind their choice in each case. However, two studies used both theoretical and purposive sampling (Lichtenstein and Williamson 2006, Andrews and Boyle 2008) and, surprisingly, two papers reported that data collection and analysis proceed simultaneously without mentioning their sampling technique (Madell and Muncer 2007, Halaweh et al. 2008).

\subsubsection{How did theoretical development continue after the core category identi?cation?}

After the initial development of concepts, further coding strategies (axial and selective coding) were followed by four of the nine studies (Daengbuppha et al. 2006, Andrews and Boyle 2008, Palka et al. 2009, Papathanassis and Knolle 2011). The coding process was explained in a range of different ways. For instance, Palka et al. (2009) described making connections between categories, while Daengbuppha et al. (2006) utilised it to relate categories with subcategories. Content analysis was used only by one study that had not followed the coding process (Lichtenstein and Williamson 2006).

\subsubsection{What was the end product of this research and how was it finally constructed?}

All of the reviewed studies summarised core and emerging categories at the end of the analysis. The recognition of an emergent core category is paramount to GT, without which a GT study might not be considered as such (Strauss and Corbin 1990, Glaser 2004, Glaser and Holton 2004). This core variable accounts for most of the variation around the problem that is the focus of the study and relates meaningfully with all other emerging categories (Glaser 1998, Glaser and Holton 2004). Their main findings were presented in tables or diagrams/models which thoroughly explained the meaning of the constructs in online/mobile customer behaviour. Only one study explained its results briefly, without clarification of how the final constructs were decided (Halaweh et al. 2008), which can be justified by the fact that it was presented at an international conference and not in a journal. Three of the nine studies argued that theoretical completeness was reached (Daengbuppha et al. 2006, Brunger and Perelli 2009, Papathanassis and Knolle 2011), while the others did not mention the notion of theoretical completeness or theoretical saturation at all.

Page 663 - ->

\subsection{Does GT contribute to existing customer behaviour theories?}

After classifying the nine studies' core and emerging categories, a comparison between them and existing customer behaviour theories was carried out to assess possible contributions of GT to online and mobile customer behaviour. As shown in Table 2, certain core or emerging categories are similar to factors already described in the existing models of TAM, TPB, IDT (and their extensions), and in some cases a combination of these. Nevertheless, new categories emerged and warrant further investigation.

Study 1 (Lichtenstein and Williamson 2006): Some of its categories were similar to TAM and TPB (usability, adoption, and convenience). The authors identified four new categories influencing consumer's adoption of Internet banking: attention, accessibility, relative advantage, and knowledge and support. 
Study 2 (Daengbuppha et al. 2006): Preconceptions in the present study were minimal. Nevertheless, the authors have taken into account assumptions from TPB (behavioural norms) or TAM (perceived value), which are apparent in their emerging categories. In addition to the existing theory, matter of choice, involvement, and interactive experience were identified.

Study 3 (Namkung et al. 2007): Some of the identified categories were similar to TAM (usability, experience). New categories were discovered as well, though, such as weborgraphics, and level of communication in understanding the web behaviour of restaurant customers.

Study 4 (Madell and Muncer 2007): A new factor - control over social interactions - was identified as influencing young people in their adoption of the Internet and mobile phones. Although the emerging categories were not presented in the paper, some similarity with the TPB model is evident in the discussion part, such as participants' behaviour and emotional situations.

Study 5 (Andrews and Boyle 2008): The present study had a satisfactory overall GT execution quality, but the authors appear to have been pre-influenced by TAM and IDT, from which they derive certain assumptions. New categories recognised include computer system invasion and mass media influence on perceptions of risk online.

Study 6 (Halaweh et al. 2008): Categories similar to TAM and TPB models were recognised (attitude, behavioural intention). In spite of this, two additional factors, security and psychological, were found.

Study 7 (Brunger and Perelli 2009): It is conceptually similar to TPB, as categories concerning behavioural control and customers' behaviour in general were discussed throughout the paper. However, five new categories were encountered: communication efficiency, search breadth, search involvement, social component, and search enthusiasm.

Study 8 (Palka et al. 2009): Some of the discovered categories of the paper were parallel with TAM (attitude, perceived usefulness, friendliness, and enjoyment) and TPB (subjective norm, intention, and behaviour) models. The category perceived cost was new in exploring customers' motivations, attitudes, and behaviours of mobile viral marketing.

Study 9 (Papathanassis and Knolle 2011): It is similar to TAM (usefulness, behavioural intention) by enhancing user perceptions for holiday purchase decisions, with further variables added: perceived accessibility, richness, macro and micro reputation, perceived factuality, neutrality, and realism.

\section{Discussion}

It was easier to know it than to explain why I know it.

It is a capital mistake to theorize before you have all the evidence. It biases the judgment.

Sherlock Holmes (Conan Doyle 1887, pp. 26-27)

We performed a critical evaluation of GT use in online/mobile customer behaviour research. Nine studies were identified with varying quality characteristics; their contribution to existing 
customer behaviour theories was possibly biased. The present study's outcome revealed the use of GT in online/mobile customer behaviour research is rudimentary, since most of the nine reviewed studies demonstrated an insufficient understanding of its methodology or a poor explanation of the research process, compromising their quality. This is evident as a number of the common misconceptions in relation to GT were identified (Tan 2010).

The presence of methodological mistakes or pitfalls in GT theory has been commented on widely (Becker 1993, Wilson and Hutchinson 1996) and traditionally involves the following GT procedures: epistemology background, theoretical sampling, coding, constant comparative method, theoretical completeness, and theoretical sensitivity. In the present study, misconceptions were obvious in theoretical sensitivity and pre-conceptualisations, the literature review, sampling, and memoing. A detailed account of these GT processes is given below, noting however that they are interconnected and performed simultaneously and should not be viewed as separate elements of GT.

Generating GT requires what Glaser (1978) has called theoretical sensitivity, a quality that combines interpersonal perceptiveness with conceptual thinking. Theoretical sensitivity includes the researchers' level of insight into the research area, how attuned they are to the subtle differences and complexity of the participants' words and actions, their ability to reconstruct meaning from the data generated with the participant, while discriminating what is relevant from what is not (Schreiber 2001, Glaser 2002a, Glaser and Holton 2004, Mills et al. 2006). Researchers can cultivate theoretical sensitivity when they continuously challenge their personal theories and biases against data, detaching them from predetermined ideas (Schreiber 2001). In the present review, papers were found to have been influenced

Page $664-->$

by existing customer behaviour theories in their selection of categories, thus indicating researchers' failure to block their pre-conceptions.

Next, treatment of the literature when GT is utilised has created conflicting ideas among researchers (Cutcliffe 2000, Martin 2006b, McCallin 2006). According to Glaser and Holton (2004), 'to undertake an extensive review of literature before the emergence of a core category violates the basic premise of GT, that theory emerges from the data not from extant theory'. This is based on the idea that literature reviews will bias GT data collection/analysis/sampling process with pre-conceptualisations (Stern 1980, Lincoln and Guba 1985, Hutchinson 1993). The timing of the literature review is decisive: once the core category has emerged, the literature review is used to integrate the theory, or it is used as data to be analysed. Three out of the nine studies reviewed conducted a literature review following core category emergence, integrating the literature into theory (Daengbuppha et al. 2006, Palka et al. 2009, Papathanassis and Knolle 2011); two studies did not produce a literature review (Madell and Muncer 2007, Halaweh et al. 2008); while the remaining four had performed a literature review before GT application, thus being pre-conceived (Lichtenstein and Williamson 2006, Namkung et al. 2007, Andrews and Boyle 2008, Brunger and Perelli 2009). Additionally, the similarities of GT results with TAM, TPB, and IDT are indicative of the presence of pre-conceptualisations.

Another misconception observed in the present review was in theoretical sampling practice and its role in theoretical completeness. An essential dictum of GT is the simultaneous nature of data collection and analysis based on constant comparisons and suspension of pre-conceptions. Only this process allows for categories to emerge and for the direction of future sampling to become apparent, thus substantiating its theoretical nature. This simultaneous process allows for category saturation, theoretical completeness, and 
ultimately theory generation (Glaser and Strauss 1967). Results of the reviewed studies showed that only three out of the nine studies used theoretical sampling, and of those three, only one referred to theoretical completeness (Daengbuppha et al. 2006).

Memoing is critical to the GT process. The researcher captures his/her analysis in memos; this includes his ideas about codes and the relationships between codes (Glaser 1998, 2012, Glaser and Holton 2004). During analysis, questions occur, gaps in the emerging theory are noticed, and preconceptions (if any) are noted and compared with the data. Thus, memos initially serve to augment analytical ideas of the primary analysis, becoming increasingly theoretical and abstract through constant comparisons, while at the same time driving theoretical sampling and enhancing theoretical sensitivity. Hence, the Glaserian statements that 'memos locate the emerging theory' and 'without using memos, the researcher is not in fact doing grounded theory' are actually quintessential to GT (Glaser 1978). Results from the reviewed studies illustrated that only two out of nine studies reported using memos, four stated using constant comparisons and six identified a core category; the remaining studies did not present the above features or it was unclear from their descriptions whether they had used them. These elements are crucial in the implementation of GT, the omission of which is attributed to research misconceptions discussed below.

Finally, GT is not presentation of raw data or routine application of formulaic techniques to data (Suddaby 2006, Tan 2010). However, it has been postulated that few published papers feature analyses driven entirely by respondents' concerns, and the terminology and theories to which papers appeal generally bear a keen resemblance to current disciplinary concerns and debates (Barbour 2001). This practice has associated GT with the epithet approving bumper sticker, invoked to confer academic respectability rather than as a helpful description of the strategy used in analysis explaining how theoretical insights have been built up (Bryman and Burgess 1994).

So, despite researchers' choice of GT, their misconceptions about the method may have biased their results, ultimately preventing theory generation. However, their systematic presence among the papers reviewed might suggest common research biases. Bias might commonly refer to prejudice, but in research it denotes the deviation from the truth (Grimes and Schulz 2002) at the expense of (possibly equally valid) alternatives. Anything biased generally is one-sided, and therefore lacks a neutral point of view. Bias can come in many forms (Grimes and Schulz 2002); we postulate that reporting, verification, and methodological biases are present. Methodological bias refers to the researcher's inadequate knowledge of the method being used. This is connected to the misconception that GT data can be analysed with prescribed canons, described above (Suddaby 2006). The second bias is verification bias. In business research, it is common to try including all results (quantitative or qualitative) under existing general sociological theories, considered as gold standards (Hammersley and Gomm 1997). Thus, the production of theory is biased by the need to retain the preconceptions of existing theories in use, instead of recognising the power of GT to generate theories. Finally, reporting bias refers to errors made in the way results are disseminated in the literature. This bias might be indicated by authors' view to present results or research design selectively, aiming to describe significant outcomes at the expense of non-significant outcomes in order to get published. It might also indicate journals' publishing policies, which tend to reject studies with negative results (McGauran et al. 2010).

\subsection{Limitations}


The findings of the present critical review are not without limitations. The quality of the instrument developed to assess the quality of grounded theories reviewed could be questioned. However, we are confident that the key tenets of

Page $665-->$

GT (constant comparison, theoretical sampling, theoretical saturation and the interchangeability of indicators, memoing, and theoretical completeness) have been adequately accounted for in the instrument. Additionally, evaluation of GT's quality was based exclusively on information presented in the studies themselves without taking external factors into consideration (i.e. word limitation from journals) that might have prevented researchers from presenting an extensive discussion about the GT process. Furthermore, because studies appeared to be preconceived, and thus focused on their professional interest rather than the interests of participants, contributions to the existing customer behaviour theory should be treated with caution.

\section{Conclusion}

In conclusion, no study followed the principles of the GT methodology due to omission of basic elements of the method or not giving clear explanation of their use (Figure 2). Therefore, results should be treated with caution from studies not applying the main elements of GT. The greatest weaknesses identified in the studies reviewed involved researchers' pre-conceptions and inadequate knowledge of the GT methodology. Thus, firm knowledge of the different versions of GT ought to precede GT application. The aim should be to understand the main concern of participants and to explain how they resolve or process their main concern. A researcher aims for theoretical completeness and conceptual integration (Glaser 1978, Murphy et al. 1998). Studies claiming to have used GT should at a minimum include evidence of simultaneous data collection and analysis; construction of analytic codes and categories from data, not from preconceived, logically deduced hypotheses; use of the constant comparative method; core category generation; advancement of theory development during each step of data collection and analysis; memo-writing; theoretical sampling; and conducting a literature review after core category emergence.

\section{Acknowledgements}

We would like to thank three anonymous reviewers, whose comments greatly improved the present paper. Aikaterini Valvi's research is supported by a scholarship from the School of Business, Economics and Informatics, Birkbeck, University of London, UK. 


\section{References}

Abdul-Muhmin, A. G. 2011. Repeat purchase intentions in online shopping: the role of satisfaction, attitude, and online retailers' performance. Journal of International Consumer Marketing, 23(1): 5-20. (doi:10.1080/08961530.2011.524571)

Ajzen, I. 1991. The theory of planned behavior. Organizational Behavior and Human Decision Processes, 50(2): 179-211. (doi:10.1016/0749-5978(91)90020-T)

Andrews, L. and Boyle, M. V. 2008. Consumers' accounts of perceived risk online and the influence of communication sources. Qualitative Market Research: An International Journal, 11(1): 59-75. (doi:10.1108/13522750810845559)

Balasubramanian, S., Peterson, R. A. and Jarvenpaa, S. L. 2002. Exploring the implications of $\mathrm{m}$-commerce for markets and marketing. Journal of the Academy of Marketing Science, 30(4): 348-361. (doi:10.1177/009207002236910)

Barbour, R. S. 2001. Checklists for improving rigour in qualitative research: a case of the tail wagging the dog?. BMJ: British Medical Journal, 322(7294): 1115-1117. (doi:10.1136/bmj.322.7294.1115)

Becker, P. H. 1993. Common pitfalls in published grounded theory research. Qualitative Health Research, 3(2): 254-260. (doi:10.1177/104973239300300207)

Belk, R. W., Wallendorf, M. and Sherry Jr, J. F. 1989. The sacred and the profane in consumer behavior: theodicy on the odyssey. Journal of Consumer Research, 16(1): 1-38. (doi:10.1086/209191)

Bhattacherjee, A. 2001. Understanding information systems continuance: an expectationconfirmation model. MIS Quarterly, 25(3): 351-370. (doi:10.2307/3250921)

Bowen, G. A. 2009. Supporting a grounded theory with an audit trail: an illustration. International Journal of Social Research Methodology, 12(4): 305-316. (doi:10.1080/13645570802156196)

Brunger, W. G. and Perelli, S. 2009. The impact of the internet on airline fares: customer perspectives on the transition to internet distribution. Journal of Revenue and Pricing Management, 8(2-3): 187-199. (doi:10.1057/rpm.2008.31)

Bryman, A. and Burgess, R. G. 1994. Analyzing qualitative data, London: Routledge. [CrossRef]

Charmaz, K. 1995. "Grounded theory". In Rethinking methods in psychology, Edited by: Smith, J. A., Harré, R. and Van Langenhove, L. 27-49. London: Sage.

Charmaz, K. 2000. "Grounded theory. Objectivist and constructivist methods.". In Sage handbook of qualitative research, 2, Edited by: Lincoln, N. K. and Denzin, Y. S. 509535. Thousands Oaks, CA: Sage.

Charmaz, K. 2006. Constructing grounded theory: a practical guide through qualitative analysis, Thousand Oaks, CA: Sage.

Chen, H. Y. and Boore, J. R.P. 2009. Using a synthesised technique for grounded theory in nursing research. Journal of Clinical Nursing, 18(16): 2251-2260. (doi:10.1111/j.13652702.2008.02684.x)

Chen, Q., Rodgers, S. and He, Y. 2008. A critical review of the e-satisfaction literature. American Behavioral Scientist, 52(1): 38-59. (doi:10.1177/0002764208321340)

Cheung, C. M.K. Online consumer behavior: a review and agenda for future research. Bled. Proceedings of 16th Bled eCommerce conference., pp.194-218.

Conan Doyle, A. 1887. A study in scarlet, London: Ward Lock.

Constantinides, E. 2004. Influencing the online consumer's behavior: the Web experience. Internet Research, 14(2): 111-126. (doi:10.1108/10662240410530835)

Corbin, J. M. and Strauss, A. 1990. Grounded theory research: procedures, canons, and evaluative criteria. Qualitative Sociology, 13(1): 3-21. (doi:10.1007/BF00988593)

Coyne, I. T. 1997. Sampling in qualitative research. Purposeful and theoretical sampling; merging or clear boundaries?. Journal of Advanced Nursing, 26(3): 623-630. (doi:10.1046/j.1365-2648.1997.t01-25-00999.x

Creswell, J. W. and Miller, D. L. 2000. Determining validity in qualitative inquiry. Theory Into Practice, 39(3): 124-130. (doi:10.1207/s15430421tip3903_2) 
Cutcliffe, J. R. 2000. Methodological issues in grounded theory. Journal of Advanced Nursing, 31(6): 1476-1484. (doi:10.1046/j.1365-2648.2000.01430.x)

Daengbuppha, J., Hemmington, N. and Wilkes, K. 2006. Using grounded theory to model visitor experiences at heritage sites: methodological and practical issues. Qualitative Market Research: An International Journal, 9(4): 367-388. (doi:10.1108/13522750610689096)

Davis, F. D., Bagozzi, R. P. and Warshaw, P. R. 1989. User acceptance of computer technology: a comparison of two theoretical models. Management Science, 35(8): 982-1003. (doi:10.1287/mnsc.35.8.982)

Dunne, C. 2011. The place of the literature review in grounded theory research. International Journal of Social Research Methodology, 14(2): 111-124. (doi:10.1080/13645579.2010.494930)

Page 666 - ->

Fishbein, M. and Ajzen, I. 1975. Belief, attitude, intention, and behavior: an introduction to theory and research, Reading, MA: Addison-Wesley.

Gephart, R. 2004. Qualitative research and the academy of management journal. Academy of Management Journal, 47(4): 454-462. (doi:10.5465/AMJ.2004.14438580)

Glaser, B. G. 1978. Theoretical sensitivity, Mill Valley, CA: Sociology Press.

Glaser, B. G. 1998. Doing grounded theory: issues and discussions, Mill Valley, CA: Sociology Press.

Glaser, B. G. 2002a. Conceptualization: on theory and theorizing using grounded theory. International Journal of Qualitative Methods, 1(2): 23-38.

Glaser, B. G. 2002b. Constructivist grounded theory?. Forum Qualitative Sozialforschung, 3(3) Article no. 12.

Glaser, B. G. 2004. Naturalist inquiry and grounded theory. Forum Qualitative Sozialforschung,, 5(1) Article no. 7.

Glaser, B. G. 2005. The impact of symbolic interaction on Grounded Theory. Grounded Theory Review, 4(2): 1-22.

Glaser, B. G. 2012. Stop. Write! writing grounded theory. Grounded Theory Review, 11(1): $2-11$.

Glaser, B. G. and Holton, J. 2004. Remodeling grounded theory. Forum Qualitative Sozialforschung, 5(1) Article no. 4.

Glaser, B. G. and Strauss, A. L. 1967. The discovery of grounded theory: strategies for qualitative research, Chicago: Aldine.

González-Teruel, A. and Abad-García, M. F. 2012. Grounded theory for generating theory in the study of behavior. Library \& Information Science Research, 34(1): 31-36. (doi:10.1016/j.lisr.2011.02.006)

Goulding, C. 2002. Grounded theory: a practical guide for management, business and market researchers., Thousand Oaks, CA: Sage.

Goulding, C. 2005. Grounded theory, ethnography and phenomenology: a comparative analysis of three qualitative strategies for marketing research. European Journal of Marketing, 39(3-4): 294-308. (doi:10.1108/03090560510581782)

Goulding, C. 2009. "Grounded theory perspectives in organizational research.". In Sage handbook of organizational research methods, Edited by: Buchanan, D. A. and Bryman, A. 381-394. Thousand Oaks, CA: Sage.

Grant, R., Clarke, R. J. and Kyriazis, E. 2007. A review of factors affecting online consumer search behaviour from an information value perspective. Journal of Marketing Management, 23(5-6): 519-533. (doi:10.1362/026725707X212801)

Grimes, D. A. and Schulz, K. F. 2002. Bias and causal associations in observational research. Lancet, 359(9302): 248-252. (doi:10.1016/S0140-6736(02)07451-2)

Halaweh, M., Fidler, C. and McRobb, S. 2008. "Security perception in e-commerce websitesA customer's perspective.". In Proceeding of IADIS international conference on ecommerce Edited by: Krishnamurthy, S. 307-310. Amsterdam, The Netherlands 
Hallberg, L. R.-M. 2006. The 'core category' of grounded theory: making constant comparisons. International Journal of Qualitative Studies on Health and Well-being, 1(3): 141-148. (doi:10.1080/17482620600858399)

Hammersley, M. and Gomm, R. 1997. Bias in social research. Sociological Research Online, 2(1): xiii-xiv. (doi:10.5153/sro.55)

Hutchinson, S. A. 1993. "Grounded theory: the method.". In Nursing research: a qualitative perspective, 2, Edited by: Munhall, P. L. and Boyd, C. A. 180-212. New York: National League for Nursing Press.

Hutchison, A. J., Johnston, L. and Breckon, J. 2011. Grounded theory-based research within exercise psychology: a critical review. Qualitative Research in Psychology, 8(3): 247272. (doi:10.1080/14780880903304527)

Jiang, P. and Rosenbloom, B. 2005. Customer intention to return online: price perception, attribute-level performance, and satisfaction unfolding over time. European Journal of Marketing, 39(1-2): 150-174. (doi:10.1108/03090560510572061)

Jones, R. and Noble, G. 2007. Grounded theory and management research: a lack of integrity?. Qualitative Research in Organizations and Management: An International Journal, 2(2): 84-103. (doi:10.1108/17465640710778502)

Kim, Y. G., Eves, A. and Scarles, C. 2009. Building a model of local food consumption on trips and holidays: a grounded theory approach. International Journal of Hospitality Management, 28(3): 423-431. (doi:10.1016/j.jjhm.2008.11.005)

Koufaris, M. 2002. Applying the technology acceptance model and flow theory to online consumer behavior. Information Systems Research, 13(2): 205-223. (doi:10.1287/isre.13.2.205.83)

Lichtenstein, S. and Williamson, K. 2006. Understanding consumer adoption of internet banking: an interpretive study in the Australian banking context. Journal of Electronic Commerce Research, 7(2): 50-66.

Lincoln, Y. S. and Guba, E. G. 1985. Naturalistic inquiry, Newbury Park, CA: Sage.

$\mathrm{Lu}, \mathrm{H}$. P. and Su, P. Y.J. 2009. Factors affecting purchase intention on mobile shopping web sites. Internet Research, 19(4): 442-458. (doi:10.1108/10662240910981399)

Madell, D. E. and Muncer, S. J. 2007. Control over social interactions: an important reason for young people's use of the Internet and mobile phones for communication?. CyberPsychology \& Behavior, 10(1): 137-140. (doi:10.1089/cpb.2006.9980)

Martin, V. B. 2005. The grounded theory bookshelf. Grounded Theory Review, 4(2): 117122.

Martin, V. B. 2006a. The postmodern turn: shall classic grounded theory take that Detour? A review essay. Grounded Theory Review, 5(2-3): 119-129.

Martin, V. B. 2006b. The relationship between an emerging grounded theory and the existing literature: four phases for consideration. Grounded Theory Review, 5(2-3): 47-50.

McCallin, A. M. 2006. Grappling with the literature in a grounded theory study. Grounded Theory Review, 5(2-3): 11-27.

McGauran, N. 2010. Reporting bias in medical research - a narrative review. Trials, 11(4): 37 (doi:10.1186/1745-6215-11-37)

Mills, J., Bonner, A. and Francis, K. 2006. The development of constructivist grounded theory. International Journal of Qualitative Methods, 5(1): 25-35.

Murphy, E. 1998. Qualitative research methods in health technology assessment: a review of the literature. Health Technology Assessment. 2(16): iii-ix. 1-274.

Namkung, Y., Shin, S.-Y. and Yang, I.-S. 2007. A grounded theory approach to understanding the Website experiences of restaurant customers. Journal of Foodservice Business Research, 10(1): 77-99. (doi:10.1300/J369v10n01_05

Ngai, E. W.T. and Gunasekaran, A. 2007. A review for mobile commerce research and applications. Decision Support Systems, 43(1): 3-15. (doi:10.1016/j.dss.2005.05.003)

Palka, W., Pousttchi, K. and Wiedemann, D. G. 2009. Mobile word-of-mouth - a grounded theory of mobile viral marketing. Journal of Information Technology, 24(2): 172-185. (doi:10.1057/jit.2008.37) 
Papathanassis, A. and Knolle, F. 2011. Exploring the adoption and processing of online holiday reviews: a grounded theory approach. Tourism Management, 32(2): 215-224. (doi:10.1016/j.tourman.2009.12.005)

Park, T., Shenoy, R. and Salvendy, G. 2008. Effective advertising on mobile phones: a literature review and presentation of results from 53 case studies. Behaviour \& Information Technology, 27(5): 355-373. (doi:10.1080/01449290600958882)

Park, Y. A. and Gretzel, U. 2007. Success factors for destination marketing Web sites: a qualitative meta-analysis. Journal of Travel Research, 46(1): 46-63. (doi:10.1177/0047287507302381)

Payne, S. 2007. "Grounded theory.". In Analysing qualitative data in psychology, Edited by: Lyons, E. and Coyle, A. 65-86. London: Sage.

Pettigrew, S. 2002. A grounded theory of beer consumption in Australia. Qualitative Market Research: An International Journal, 5(2): 112-122. (doi:10.1108/13522750210423814)

Page 667 - ->

Ranaweera, C., McDougall, G. and Bansal, H. 2005. A model of online customer behavior during the initial transaction: moderating effects of customer characteristics. Marketing Theory, 5(1): 51-74. (doi:10.1177/1470593105049601)

Ridley, D. 2008. The literature review, London: Sage.

Rogers, E. M. 1962. Diffusion of innovations, Glencoe: Free Press.

Rose, S., Hair, N. and Clark, M. 2011. Online customer experience: a review of the business-to-consumer online purchase context. International Journal of Management Reviews, 13(1): 24-39. (doi:10.1111/j.1468-2370.2010.00280.x)

Schreiber, R. S. 2001. "The 'how to' of grounded theory: avoiding the pitfalls.". In Using grounded theory in nursing, Edited by: Schreiber, R. S. and Stern, P. N. 55-83. New York: Springer.

Stern, P. N. 1980. Grounded theory methodology: its uses and processes. Journal of Nursing Scholarship, 12(1): 20-23. (doi:10.1111/j.1547-5069.1980.tb01455.x)

Strauss, A. and Corbin, J. 1990. Basics of qualitative research: grounded theory procedures and techniques., Newbury Park, CA: Sage.

Strauss, A. and Corbin, J. 1994. "Grounded theory methodology: an overview.". In Handbook of qualitative research, Edited by: Denzin, N. K. and Lincoln, Y. S. 273-285. London: Sage.

Suddaby, R. 2006. From the editors: what grounded theory is not. Academy of Management Journal, 49(4): 633-642. (doi:10.5465/AMJ.2006.22083020)

Tan, J. 2010. Grounded theory in practice: issues and discussion for new qualitative researchers. Journal of Documentation,, 66(1): 93-112. (doi:10.1108/00220411011016380)

Valvi, A. C. and Fragkos, K. C. 2012. Critical review of the e-loyalty literature: a purchasecentred framework. Electronic Commerce Research, 12(3): 331-378. (doi:10.1007/s10660-012-9097-5)

Wagner, S. M., Lukassen, P. and Mahlendorf, M. 2010. Misused and missed use - grounded theory and objective hermeneutics as methods for research in industrial marketing. Industrial Marketing Management, 39(1): 5-15. (doi:10.1016/j.indmarman.2008.05.007)

Wallace, M. and Wray, A. 2011. Critical reading and writing for postgraduates, 2, London: Sage.

Wilson, H. S. and Hutchinson, S. A. 1996. Methodologic mistakes in grounded theory. Nursing Research, 45(2): 122-124. (doi:10.1097/00006199-199603000-00012)

$\mathrm{Wu}, \mathrm{X}$. 2010. A review of mobile commerce consumers' behaviour research: consumer acceptance, loyalty and continuance (2000-2009). International Journal of Mobile Communications, 8(5): 528-560. (doi:10.1504/IJMC.2010.034936) 
Yuan, Y. and Zhang, J. J. 2003. Towards an appropriate business model for $\mathrm{m}$-commerce. International Journal of Mobile Communications, 1(1): 35-56. (doi:10.1504/IJMC.2003.002459) 